\title{
Regulation of Renal Fibrosis by Macrophage Polarization
}

\author{
Bixia Pan ${ }^{a}$ Guohui Liu ${ }^{a}$ Zongpei Jiang ${ }^{b}$ Dongwen Zheng ${ }^{a}$ \\ aDepartment of Nephrology, Dongguan People's Hospital, Dongguan, 'Department of Nephrology, \\ the Sixth Affiliated Hospital of Sun Yat-sen University, Guangzhou, China
}

\section{Key Words}

Macrophages - Unilateral ureteral obstruction (UUO) - Renal fibrosis • Epithelial-tomesenchymal transition (EMT) • Transforming growth factor $\beta 1$ (TGF $\beta 1$ ) • Bone morphogenic protein 7 (BMP7) $\bullet$ E-cadherin $\bullet \alpha$-smooth muscle actin $(\alpha-S M A)$

\begin{abstract}
:
Background/Aims: Since renal fibrosis always predisposes end-stage renal disease, elucidation of the molecular mechanisms that underlie the progression of renal fibrosis may substantially improve the understanding and treatment for renal failure. Previous studies have highlighted an important counteraction between transforming growth factor $\beta 1$ (TGF $\beta 1$ ) and bone morphogenic protein 7 (BMP7) in the epithelial-to-mesenchymal transition (EMT) of renal tubular epithelial cells during chronic renal injury. Macrophages are also believed to play a critical role in renal fibrosis. However, the relationship between macrophages and EMT is unknown. Methods: Here, we used a mouse unilateral ureteral obstruction (UUO) model to address to these questions, and analyzed macrophage and its subpopulations purified by flow cytometry. Results: We found that the recruited macrophages are polarized to a M2 subtype after renal injury. M2 macrophages released high levels TGF $\beta 1$ to suppress BMP7 to enhance EMT-induced renal fibrosis. Depletion of M2 macrophages, but not of M1 macrophages, specifically inhibited EMT, and subsequently the renal fibrosis. Adoptive transplantation of M2 macrophages deteriorated renal fibrosis. Conclusion: Thus, our study highlights M2 macrophages as a critical target for treating renal fibrosis.
\end{abstract}




\section{Introduction}

Renal failure often results from obstructive nephropathy, although the exact cellular and molecular mechanisms have not been elucidated. Urinary tract obstruction and tubular dilatation lead to tubular apoptosis and local macrophage infiltration, resulting in increases in interstitial fibroblasts through both proliferation of resident fibroblasts and epithelialto-mesenchymal transition (EMT) of renal tubular cells. During EMT, tubular epithelial cells acquire a mesenchymal phenotype with enhanced migratory capacity, and then these transformed cells migrate from the renal tubular microenvironment into the interstitial space, as a major contributor to the pathogenesis of renal fibrosis. Meanwhile, fibroblasts also transform into myofibroblasts that generate excess deposition of extracellular matrix in response to cytokines, chemokines and other signaling molecules [1-4].

Two TGF $\beta$ superfamily members [5-7], bone morphogenic protein 7 (BMP7) and transforming growth factor $\beta 1$ (TGF $\beta 1$ ), are critical regulators of renal epithelial cell plasticity in the kidney. TGF $\beta 1$ is a potential inducer of EMT, while the effect of TGF $\beta 1$ is efficiently inhibited by BMP7 [1-4, 8-11]. The crosstalk between TGF $\beta 1$ and BMP7 coordinates the repair and recovery of the injured kidney [12-14]. The Unilateral ureteral obstruction (UU0) model is a well-established experimental obstructive nephropathy model for generating progressive renal fibrosis in rodents $[15,16]$.

Macrophages play a substantial role in the pathogenesis of renal fibrosis, although the major focuses of previous studies are their functions in dead cells and debris removal, which are typical features for the classically activated macrophages (also called M1 macrophages) [17-20]. Besides M1 macrophages, there are also the alternatively activated macrophages (also called M2 macrophages), which mediate humoral immunity and tissue repair [2125]. Of note, M2 macrophages and their effects on kidney recovery after UUO have not been described before.

In the current study, we examined the relationship between macrophage polarization (M1/M2 ratio) and the development of renal fibrosis after UUO. We found that the recruited macrophages are polarized from F4/80-positive, CD163-negative M1 macrophages to F4/80-positive, CD163-positive M2 macrophages after UUO. Moreover, M2 macrophages released high levels of TGF $\beta 1$ to contradict BMP7 and facilitate EMT-induced renal fibrosis. M2 macrophage depletion, but not M1 macrophage depletion, specifically inhibited EMT, and subsequently inhibited renal fibrosis. Adoptive transplantation of M2 macrophages increased the features of renal fibrosis.

\section{Materials and Method}

\section{Animal handling}

Protocols for animal experimentation and maintenance were approved by the Animal Ethics Committee at Department of Nephrology of Dongguan People's Hospital. Male Balb/c mice of 10 weeks of age received left ureteral ligation with 6-gauge silk sutures. The UUO-treated mice were then randomly grouped, treated and analyzed after UUO. Intravenous injection of $200 \mu$ clodronate-liposomes (Clodronateliposome, Netherland) every 2 days were performed after UUO, to eliminate macrophages. Control mice received injections of liposome of the same amount and volume. For the adoptive M2 macrophage transplantation, purified CD163+F4/80+ M2 macrophages were transplanted under the kidney capsule for one week before final analyses.

Kidney digestion, analysis and isolation of macrophage subtypes by flow cytometry

The mouse kidney was chopped into $2 \mathrm{~mm}$-diameter pieces, and then digested with $45 \mathrm{mg} / \mathrm{dl}$ collagenase (Sigma, St Louis, USA) for 35 minutes in a $37{ }^{\circ} \mathrm{C}$ shaker at $180 \mathrm{rpm}$. The digests were then passed through a $40 \mathrm{~nm}$ filter, incubated with APC-conjugated F4/80 antibody and/or FITC-conjugated CD163 (Serotec, St Louis, USA), and then subjected to flow cytometry analyses/sorting. 
Histology and quantification of degree of renal fibrosis

Sampled kidneys were fixed in $10 \%$ formalin, embedded, and then sectioned at $4 \mu \mathrm{m}$ thickness. Masson trichrome staining was then performed for evaluation of renal fibrosis. Each sample was performed based on 25 randomly selected fields per section, which were examined under $\times 400$ magnification for assessment of the degree of renal fibrosis, as the ratio of the positive stain area to that of the whole area.

Western Blot

Kidneys or isolated macrophage subtypes were homogenized in RIPA lysis buffer (1\% NP40, 0.1\% SDS, $100 \mu \mathrm{g} / \mathrm{ml}$ phenylmethylsulfonyl fluoride, 0.5\% sodium deoxycholate, in PBS) for protein extraction. Primary antibodies for Western Blot are anti-alpha-smooth muscle actin (a-SMA), anti-E-cadherin, antiTGF $\beta 1$, anti-BMP7 and anti- $\alpha$-tubulin (protein loading control) polyclonal antibodies from Santa Cruz Biotechnology (Santa Cruz, CA, USA). Secondary antibody is HRP-conjugated anti-rabbit (Jackson Labs, CA, USA). Representative images from 5 repeats were shown in the figures. $\alpha$-tubulin was used as a protein loading control.

\section{Quantitative RT-PCR (RT-qPCR)}

RNA was extracted from kidney or sorted macrophages with Trizol (Invitrogen, St Louis, USA), or RNAeasy kit (Qiagen, Hilden, Germay) and used for cDNA synthesis with a reverse transcription kit from Qiagen. Quantitative PCR were performed in duplicates with QuantiTect SYBR Green PCR Kit (Qiagen). All the primers were purchased from Qiagen. Values of gene expression were normalized against $\alpha$-tubulin, and then compared with control values to calculate fold changes.

Statistics

All data were statistically analyzed using one-way ANOVA with a Bonferoni correction. All values are depicted as mean \pm standard deviation from 5 individuals and are considered significant if $\mathrm{p}<0.05$.

\section{Results}

\section{Macrophage polarization after UUO}

UUO is a well-established rodent model for progressive renal fibrosis in humans. Although macrophages have been shown to play an essential role in the progression of UUOinduced renal fibrosis, the exact mechanism remains largely unknown. Since macrophages have two subtypes (M1 and M2 macrophages) that are responsible for various biological functions, we thus examined the changes in macrophage subtypes after UUO.

We performed UUO on mice. At 3 days, 5 days, 7 days or 10 days after UUO, we analyzed macrophages by flow cytometry using a pan-macrophage marker F4/80 and a specific M2macrophage marker, CD163 (Fig. 1A). We then quantified the ratio of M1/M2 macrophages, and we found that the F4/80-positive macrophages in the injured kidney were mainly CD163negative M1 macrophages at 3 days after UUO. However, the ratio of M1/M2 macrophages continuously decreased since then and most F4/80-positive macrophages in the injured kidney appeared to be CD163-positive M2 macrophages by day 10 after UUO (Fig. 1B). These data suggest that the polarization of macrophages to an M2 subtype occurred during this period.

Macrophage polarization resulted in reversal of the TGF $\beta 1 / B M P 7$ ratio in the kidney

BMP7 and TGF $\beta 1$ are two most important molecular regulators for EMT of renal epithelial cells in the kidney, in which TGF $\beta 1$ is a potential inducer of EMT and BMP7 counteracts the effects of TGF $\beta 1$. Thus, we examined the relationship between macrophage polarization and the changes in TGF $\beta 1$ and BMP7 levels in the injured kidney after UUO. We measured the transcripts (Fig. 2A-B) and proteins (Fig. 2C) of TGF $\beta 1$, BMP7, and two markers for EMT, E-cadherin (an epithelial cell marker) and $\alpha$-SMA (a mesenchymal cell marker) in the kidney. We found that the TGF $\beta 1$ and $\alpha$-SMA levels increased continuously from 3 days 
Fig. 1. Macrophage polarized after UUO. (A) UUO was performed on mice. At 3 days or 10 days after UUO, infiltrated macrophages in the kidney were analyzed by flow cytometry. Representative flow chart is shown. (B) Quantification of the ratio of M1/M2 macrophages at day 3, 5, 7, 10 and 14 days after UUO by flow cytometry. *: $\mathrm{p}<0.05$.
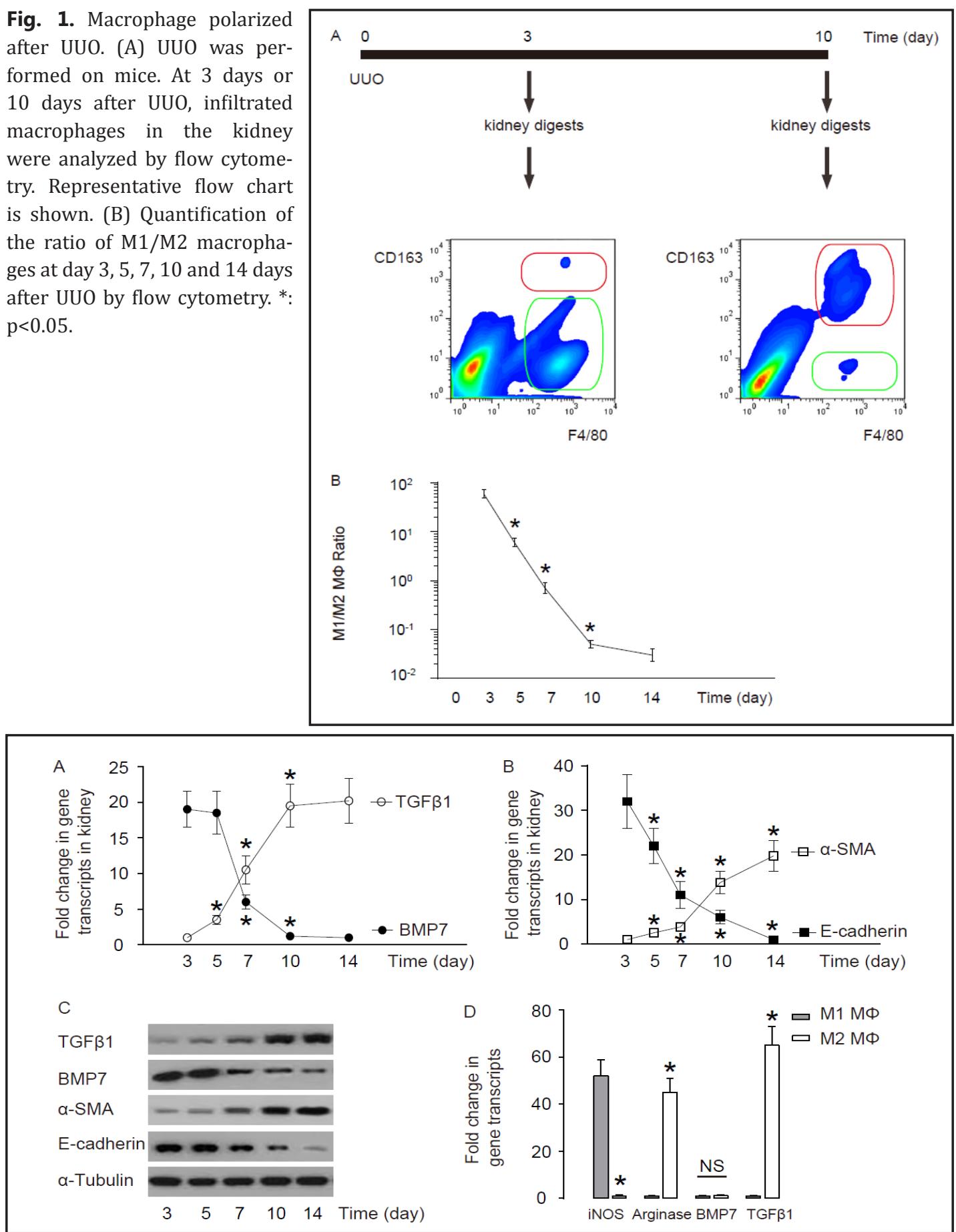

Fig. 2. Macrophage polarization and changes in TGF $\beta 1$ and BMP7 after UUO. (A) Transcript levels of TGF $\beta 1$ and BMP7 in the kidney were measured at different time points after UUO. (B) Transcript levels of E-cadherin and $\alpha$-SMA in the kidney were measured at different time points after UUO. (C) Proteins were analyzed by Western blot at different time points after UUO. $\alpha$-tubulin was used as a loading control. (D) M1 and M2 macrophages were purified from the kidney 7 days after UUO for analyses of transcript levels for TGF $\beta 1$, BMP7, iNOS and Arginase. *: p<0.05. NS: non-significant.

after UUO to 14 days after UUO, while BMP7 and E-cadherin expression decreased (Fig. 2AC), correlated with the macrophage polarization during this period. 
ig. 3. Elimination of $\mathrm{M} 2$, but not M1 macrophages, ameliorated renal fibrosis. (A) A single injection of clodronate at the same time of UUO (Day 0) to eliminate M1 macrophages. Multiple injections of clodronate every 2 days (day 4, day 6 and day8 after UUO) to eliminate M2 macrophages. (BD) The degree of renal fibrosis in Masson-stained sections at day10. (B) Changes in E-cadherin and $\alpha$-SMA mRNA levels in the kidney at D10. (C) Quantification of the degree of renal fibrosis by Masson staining. (D) Representative images for Masson-trichrome staining. *: $\mathrm{p}<0.05$. NS: non-significant. Scale bar is $50 \mu \mathrm{m}$.
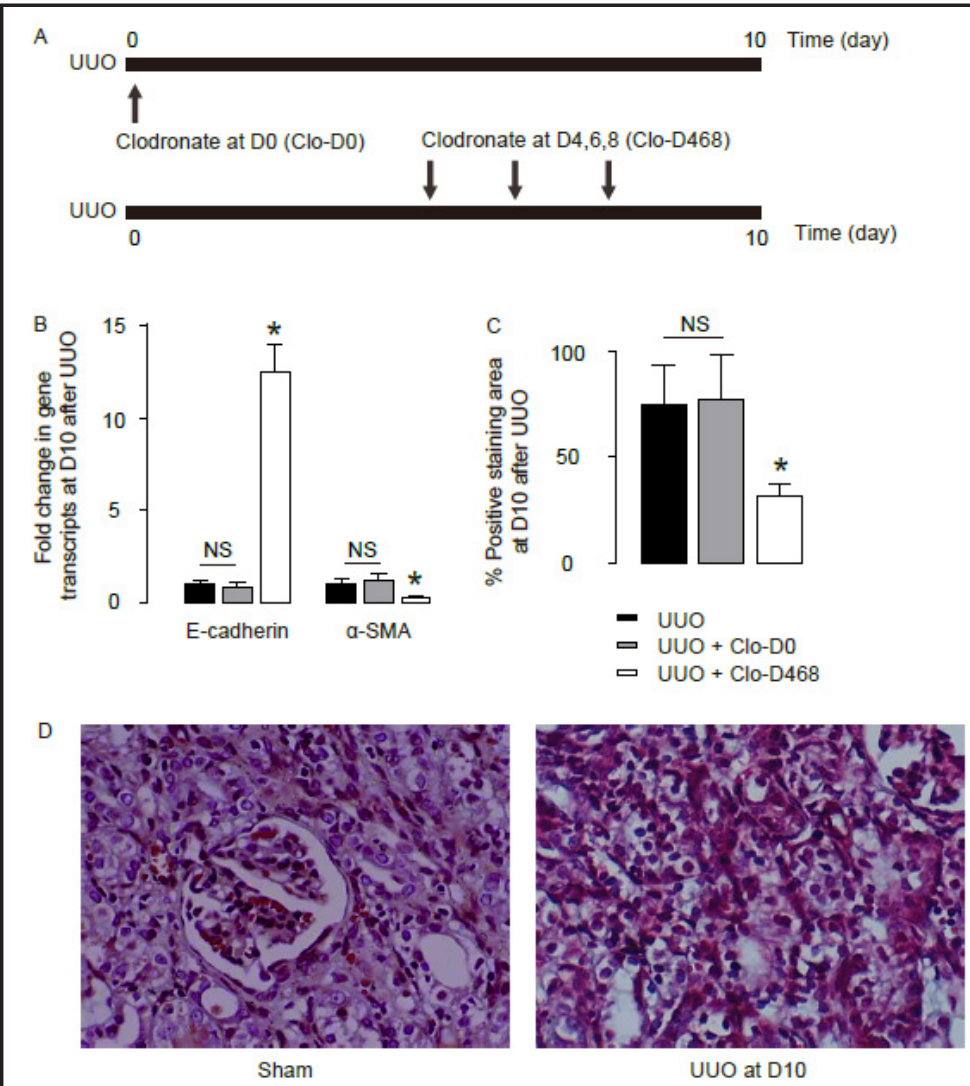

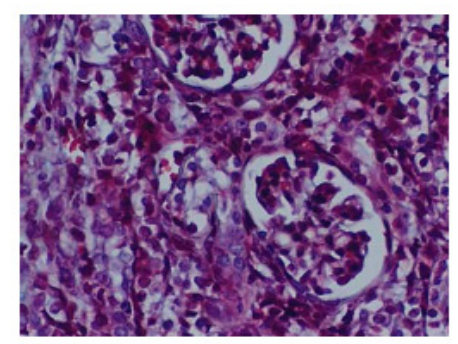

$\mathrm{UUO}+\mathrm{Clo}-\mathrm{D} 0$ at D10

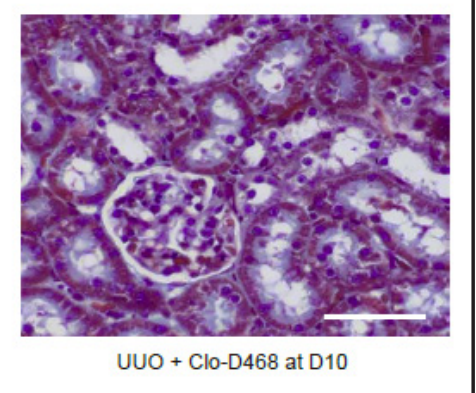

To figure out the contribution of macrophages to TGF 31 and BMP7, we isolated M1 and M2 macrophages from the kidney 7 days after UUO (Fig. 1B). Arginase (predominantly expressed by M2 macrophages) and iNOS (predominantly expressed by M1 macrophages) were used to confirm the purity of these macrophage subpopulations (Fig. 2D). Essentially no BMP7 were detected in both M1 and M2 macrophages, suggesting that macrophages do not produce BMP7 after UUO. BMP7 should be mainly produced by kidney epithelial cells [12-14]. However, although little TGF $\beta 1$ was detected in M1 macrophages, extremely high levels of TGF $\beta 1$ were detected in M2 macrophages (Fig. 2D). These data suggest that M2 macrophages may be a major contributor of TGF $\beta 1$ in the injured kidney, and M2 macrophages may play a critical role in the development of renal fibrosis.

\section{Deletion of M2 macrophages attenuated renal fibrosis by UUO}

To examine the effects of macrophage polarization on UUO-induced renal fibrosis, we used clodronate to chemically deplete the macrophages. Clodronate is a hydrophilic molecule and is packaged in a liposome to allow internalization and accumulation of clodronate in a macrophage after ingestion. Accumulation of cellular clodronate induces apoptosis of the macrophage. Neither the liposome nor clodronate are toxic to non-phagocytic cells, resulting in specific targeting and elimination of macrophages [26]. 
Fig. 4. Adoptive transfer of M2 macrophages induced features of renal fibrosis. Transplantation of $10^{6}$ CD163+F4/80+ M2 macrophages isolated from a UUO-treated kidney under the kidney capsule of recipient mice induced features of renal fibrosis.

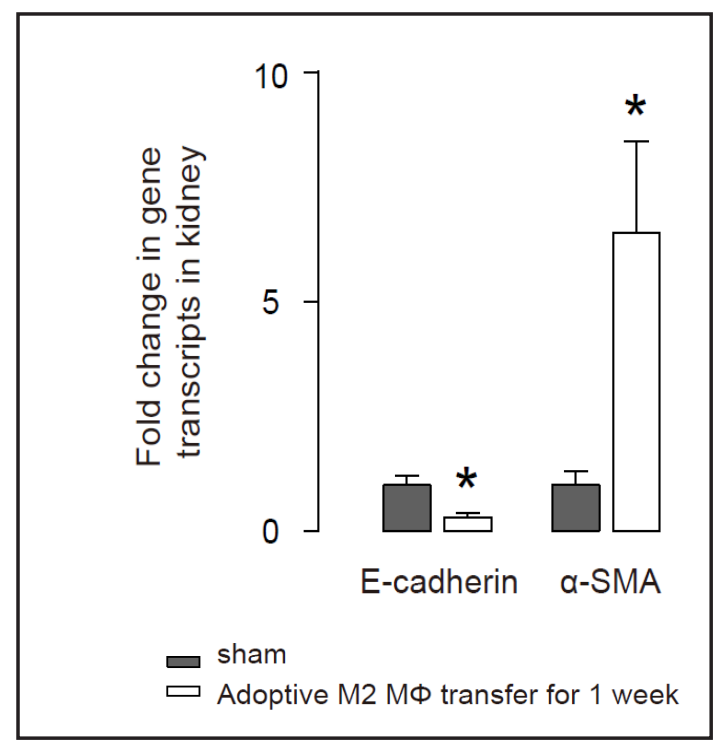

To selectively deplete M1 or M2 macrophages, we either gave a single injection of clodronate at the same time of UUO (Day 0) to eliminate M1 macrophages, or gave multiple injections of clodronate every 2 days (day 4, day 6 and day8 after UUO) to eliminate M2 macrophages (Fig. 3A). The half-life of clodronate-liposome is pretty short [25, 27-29], resulting in little effect on a later recruitment of M2 macrophages by a single injection at Day 0 . Thus, analyses on the kidney at day 10 after UUO allow us to examine the specific effects of removal of either M1 macrophages or M2 macrophages.

We found that removal of M1 macrophages had no effects on the levels of E-cadherin and $\alpha$-SMA in the kidney (Fig. 3B), and the levels of renal fibrosis (Fig. 3C-D), suggesting that M1 macrophages did not potentially contribute to the formation of renal fibrosis. However, M2 macrophage depletion by clodronate substantially mitigated the formation of renal fibrosis (Fig. 3C-D), possibly through decreased TGF 31 (Fig. 3B). To further confirm the role of M2 macrophages in the process of renal fibrosis, $10^{6} \mathrm{CD} 163+\mathrm{F} 4 / 80+\mathrm{M} 2$ macrophages isolated from UUO-treated kidney were transplanted under the kidney capsule of mice without UUO, which potentially induced features of renal fibrosis (Fig. 4).

\section{Discussion}

The molecular basis of renal fibrosis is critical for establishing novel therapeutic strategies for the prevention or treatment of progressive kidney diseases. TGF $\beta 1$ induces renal tubular epithelial cell EMT, while BMP7 counteracts TGF $\beta 1$-induced EMT $[12,14,30]$. Moreover, the macrophages are known to play essential roles in the development of renal fibrosis, but the present concerns on macrophages were mostly limited to their roles as phagocytes to clean tissue debris $[10,18,31]$.

Here we used a mouse UUO model to study the participation of macrophages in the development of renal fibrosis. We showed that the infiltrated macrophages were recruited to the inflammation milieu and appeared to be mainly M1 macrophages at beginning. However, these M1 macrophages were then polarized into M2 macrophages, suggesting functional adaptation. We used specific a specific M2 macrophage marker CD163 to differentiate M1 and M2 macrophages by flow cytometry. Previous studies have used CD206, CD301 and others but essentially reached similar results.

Here, we found that the polarization of macrophages occurred shortly after UUO, since the M1/M2 ratio completely inversed from day 3 to day 10 after UUO. These data demonstrate that the M1 macrophage-mediated phagocytosis may be only responsible for removal of 
dead cells and tissue debris at the very early stage of kidney injury after UUO. Later on, M2 macrophages appear to be the major type of macrophages and we were prompted to study their contribution to renal fibrosis.

We detected TGF $\beta 1$ and $\alpha$-SMA upregulation and polarization of during kidney fibrosis is correlated with appearance of M2 macrophages and upregulation. Since myofibroblasts have been previously shown to produce TGF $\beta 1$, we then analyzed levels of TGF $\beta 1$ in the FAC sorted myofibroblasts and compared to the levels of TGF $\beta 1$ in M2 macrophages. We found that the levels of TGF $\beta 1$ in myofibroblasts were significantly lower than those in M2 macrophages and the number of myofibroblasts was significantly lower than M2 macrophages, suggesting that the major sources for TGF $\beta 1$ in the injured kidney are M2 macrophages, rather than myofibroblasts.

We then used a well-established clodronate to specially eliminate macrophages at different time points after UUO. Interestingly, we found that elimination of M2 macrophages after UUP, but not elimination of M1 macrophages after UUO, specifically alleviated the features of renal fibrosis. Therefore, our data highlight a pivotal role of M2 macrophages in the progression of renal fibrosis, possibly through their specific expression of high levels of TGF $\beta 1$. Our data also indicate that modulation of macrophage polarization may be a promising treatment for renal fibrosis.

\section{Disclosure Statement}

The authors have declared that no competing interests exist.

\section{Reference}

1 Ma LJ, Yang H, Gaspert A, Carlesso G, Barty MM, Davidson JM, Sheppard D, Fogo AB: Transforming growth factor-beta-dependent and -independent pathways of induction of tubulointerstitial fibrosis in beta6(-/-) mice. Am J Pathol 2003;163:1261-1273.

-2 Sato M, Muragaki Y, Saika S, Roberts AB, Ooshima A: Targeted disruption of tgf-beta1/smad3 signaling protects against renal tubulointerstitial fibrosis induced by unilateral ureteral obstruction. J Clin Invest 2003;112:1486-1494.

- 3 Goto Y, Manabe N, Uchio-Yamada K, Yamaguchi-Yamada M, Inoue N, Yamamoto Y, Ogura A, Nagano N, Miyamoto H: Augmented cytoplasmic smad4 induces acceleration of tgf-beta1 signaling in renal tubulointerstitial cells of hereditary nephrotic icgn mice with chronic renal fibrosis; possible role for myofibroblastic differentiation. Cell Tissue Res 2004;315:209-221.

-4 Hwang M, Kim HJ, Noh HJ, Chang YC, Chae YM, Kim KH, Jeon JP, Lee TS, Oh HK, Lee YS, Park KK: Tgf-beta1 sirna suppresses the tubulointerstitial fibrosis in the kidney of ureteral obstruction. Exp Mol Pathol 2006;81:48-54.

5 Padua D, Massague J: Roles of tgfbeta in metastasis. Cell Res 2009;19:89-102.

6 Massague J, Chen YG: Controlling tgf-beta signaling. Genes Dev 2000;14:627-644.

7 Xiao X, Wiersch J, El-Gohary Y, Guo P, Prasadan K, Paredes J, Welsh C, Shiota C, Gittes GK: Tgfbeta receptor signaling is essential for inflammation-induced but not beta-cell workload-induced beta-cell proliferation. Diabetes 2013;62:1217-1226.

-8 Liu FY, Li XZ, Peng YM, Liu H, Liu YH: Arkadia-smad7-mediated positive regulation of tgf-beta signaling in a rat model of tubulointerstitial fibrosis. Am J Nephrol 2007;27:176-183.

-9 Wang Y, Zhang Z, Shen H, Lu Y, Li H, Ren X, Wu G: Tgf-beta1/smad7 signaling stimulates renal tubulointerstitial fibrosis induced by aai. J Recept Signal Transduct Res 2008;28:413-428.

10 Tamura M, Aizawa R, Hori M, Ozaki H: Progressive renal dysfunction and macrophage infiltration in interstitial fibrosis in an adenine-induced tubulointerstitial nephritis mouse model. Histochem Cell Biol 2009;131:483-490. 
11 Yeh YC, Wei WC, Wang YK, Lin SC, Sung JM, Tang MJ: Transforming growth factor-\{beta\}1 induces smad3dependent $\{$ beta\}1 integrin gene expression in epithelial-to-mesenchymal transition during chronic tubulointerstitial fibrosis. Am J Pathol 2010;177:1743-1754.

12 Sugimoto H, Yang C, LeBleu VS, Soubasakos MA, Giraldo M, Zeisberg M, Kalluri R: Bmp-7 functions as a novel hormone to facilitate liver regeneration. FASEB J 2007;21:256-264.

13 Zeisberg M: Bone morphogenic protein-7 and the kidney: Current concepts and open questions. Nephrol Dial Transplant 2006;21:568-573.

14 Zeisberg M, Hanai J, Sugimoto H, Mammoto T, Charytan D, Strutz F, Kalluri R: Bmp-7 counteracts tgf-beta1induced epithelial-to-mesenchymal transition and reverses chronic renal injury. Nat Med 2003;9:964-968.

15 Manucha W: Biochemical-molecular markers in unilateral ureteral obstruction. Biocell 2007;31:1-12.

16 Wen JG: Partial unilateral ureteral obstruction in rats. Neurourol Urodyn 2002;21:231-250.

17 Belmiro CL, Goncalves RG, Kozlowski EO, Werneck AF, Takyia CM, Leite-Jr M, Pavao MS: Dermatan sulfate reduces monocyte chemoattractant protein 1 and tgf-beta production, as well as macrophage recruitment and myofibroblast accumulation in mice with unilateral ureteral obstruction. Braz J Med Biol Res 2011;44:624-633.

18 Sung SA, Jo SK, Cho WY, Won NH, Kim HK: Reduction of renal fibrosis as a result of liposome encapsulated clodronate induced macrophage depletion after unilateral ureteral obstruction in rats. Nephron Exp Nephrol 2007;105:e1-9.

19 Naruse T, Yuzawa Y, Akahori T, Mizuno M, Maruyama S, Kannagi R, Hotta N, Matsuo S: P-selectindependent macrophage migration into the tubulointerstitium in unilateral ureteral obstruction. Kidney Int 2002;62:94-105.

20 Takeda A, Fukuzaki A, Kaneto H, Ishidoya S, Ogata Y, Sasaki T, Konda R, Sakai K, Orikasa S: Role of leukocyte adhesion molecules in monocyte/ macrophage infiltration in weanling rats with unilateral ureteral obstruction. Int J Urol 2000;7:415-420.

-21 Gordon S: Alternative activation of macrophages. Nat Rev Immunol 2003;3:23-35.

-22 Gordon S, Martinez FO: Alternative activation of macrophages: Mechanism and functions. Immunity 2010;32:593-604.

-23 Geissmann F, Manz MG, Jung S, Sieweke MH, Merad M, Ley K: Development of monocytes, macrophages, and dendritic cells. Science 2010;327:656-661.

24 Ricardo SD, van Goor H, Eddy AA: Macrophage diversity in renal injury and repair. J Clin Invest 2008;118:3522-3530.

25 Xiao X, Gaffar I, Guo P, Wiersch J, Fischbach S, Peirish L, Song Z, El-Gohary Y, Prasadan K, Shiota C, Gittes GK: M2 macrophages promote beta-cell proliferation by up-regulation of smad7. Proc Natl Acad Sci U S A 2014;111:E1211-1220.

-26 van Rooijen N, Bakker J, Sanders A: Transient suppression of macrophage functions by liposomeencapsulated drugs. Trends Biotechnol 1997;15:178-185.

27 van Rooijen N, van Nieuwmegen R: Elimination of phagocytic cells in the spleen after intravenous injection of liposome-encapsulated dichloromethylene diphosphonate. An enzyme-histochemical study. Cell Tissue Res 1984;238:355-358.

-28 Plosker GL, Goa KL: Clodronate. A review of its pharmacological properties and therapeutic efficacy in resorptive bone disease. Drugs 1994;47:945-982.

29 Kanis JA, McCloskey EV: Clodronate. Cancer 1997;80:1691-1695.

30 Wells RG: Bmp-7: A new tgf-beta family member takes the stage in colitis treatment. Inflamm Bowel Dis 2004;10:169.

31 Shen B, Liu X, Fan Y, Qiu J: Macrophages regulate renal fibrosis through modulating tgfbeta superfamily signaling. Inflammation 2014;37:2076-2084. 